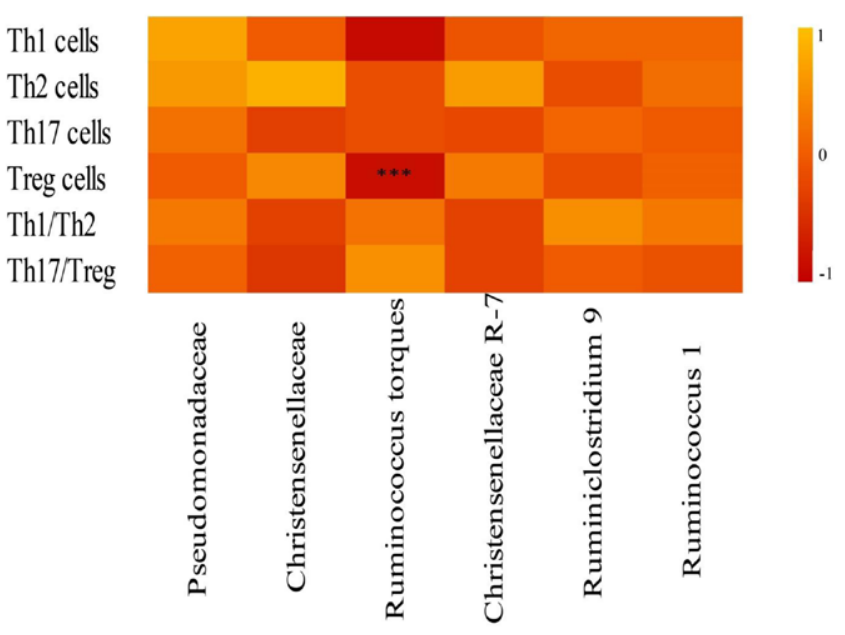

Figure 2. A heatmap shows the correlation between the intestinal microbiota and CD4+T cells in patients with RA, and Ruminococcus torques at the genus level was negative related with Treg cells. (Colors indicate the Spearman rank correlation, ${ }^{\star \star \star} \mathrm{P}<0.001$ ).

Disclosure of Interests: None declared

DOI: 10.1136/annrheumdis-2021-eular.2783

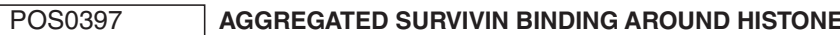 H3 EPIGENETIC MODIFICATIONS IN RISK LOCI ASSOCIATED WITH RHEUMATOID ARTHRITIS}

V. Chandrasekaran ${ }^{1}$, G. Katona ${ }^{2}$, M. I. Bokarewa ${ }^{1}$, K. M. Andersson ${ }^{1}$, M. C. Erlandsson ${ }^{1}$, M. Jensen ${ }^{2}$, N. Oparina ${ }^{1}$, A. Damdimopoulos ${ }^{3}$. ${ }^{1}$ Gothenburg University, Department of Rheumatology, Gothenburg, Sweden;

${ }^{2}$ Gothenburg University, Department of Chemistry and Molecular Biology, Gothenburg, Sweden; ${ }^{3}$ Karolinska Institute, Department of Bioscience and Nutrition, Huddinge, Sweden

Background: Survivin is an integral part of the Chromosomal Passenger Complex (CPC) which plays a vital role in mitosis. Experiments have demonstrated that survivin can physically bind to DNA. Crystallographic studies show that survivin binds to Threonine-3 of histone H3. In patients with autoimmune diseases, increased survivin expression contributes to an aggravated disease phenotype. Thus, functional, and mechanistic data point to a potential chromatin regulatory role for survivin, possibly in combination with the established gene regulatory function carried out by histone epigenetic modifications (EM)

Objectives: The objective of the study was to analyse the co-localization of chromatin bound survivin with three histone $\mathrm{H} 3$ epigenetic modifications - acetylated lysine 27 (K27ac) and trimethylated lysine 4 (K4me3) and lysine-27 (K27me3). The second objective was to analyse if survivin-bound DNA sequences overlapped with sequences in the vicinity of 106 GWAS SNPs that are associated with a risk of developing rheumatoid arthritis (RA).

Methods: Chromatin from CD4 T cells of 14 female subjects was immunoprecipitated with survivin antibodies and each of the histone $\mathrm{H} 3$ antibodies, and coupled with sequencing (ChIPseq, Hiseq2000, Illumina). After mapping the annotations of sequenced regions to the human reference genome hg38, enriched peaks were identified through Homer software. The identified survivin ChIP peaks were analysed for colocalization with peaks of the three histone $\mathrm{H} 3 \mathrm{EMs}$ and with RA risk loci, using the Bioconductor package 'ChIPPeakAnno' through RStudio.

Results: Among the total of $~ 13,000$ individual survivin ChIP-peaks, 33\% colocalized with histone $\mathrm{H} 3 \mathrm{EM}$ peaks. The overlapping peaks show a linear increase in average peak size compared with the peaks showing no colocalization with any $\mathrm{H} 3$ EM peak. A maximum of 5.5 -fold increase in average peak size was observed when survivin bound peaks overlap with peaks of all three H3 EMs. A major proportion (86\%) of top RA risk SNPs was associated with either binding of survivin or $\mathrm{H} 3 \mathrm{EMs}$. In this subset, $63 \%$ of RA risk SNPs were found within an area of 100 kilobases from survivin ChIP-peaks, with preferential enrichment of high-scoring peaks when survivin colocalizes with all $3 \mathrm{H} 3 \mathrm{EMs}$. Survivin was bound to risk SNPs annotated to, among others, the major immunological genes CD83, IRF4, CD28, ICOS and IL2RA
Conclusion: This study presents experimental evidence that survivin binding to DNA preferentially occurred in regions with high density of histone EMs. The increased aggregation of survivin around histone $\mathrm{H} 3 \mathrm{EMs}$ point to its potential regulatory function in gene transcription. Since regions around RA risk SNPs overlap with survivin peaks, survivin's nuclear function could have immunologically important effects in mechanisms of autoimmune diseases.

Disclosure of Interests: None declared

DOI: 10.1136/annrheumdis-2021-eular.3212

\section{POS0398 \\ ADIPONECTIN INDUCES SYNOVIAL ANGIOGENESIS IN RHEUMATOID ARTHRITIS THROUGH METABOLIC REMODELING}

L. Wang ${ }^{1}$, W. Tan ${ }^{1}$, F. Wang ${ }^{2}$, M. Zhang ${ }^{1} .{ }^{1}$ The First Affiliated Hospital of Nanjing Medical University, Department of Rheumatology, Nanjing, China; ${ }^{2}$ The First Affiliated Hospital of Nanjing Medical University, Department of Cardiology, Nanjing, China

Background: Our team have previously reported that Adiponectin correlates well with synovial inflammation and progressive bone erosion in rheumatoid arthritis (RA). Angiogenesis is another important part, which plays a critical role in the pathogenesis of RA.

Objectives: We hypothesized that adiponectin induces synovial angiogenesis in RA.

Methods: Single-cell RNA sequencing (scRNA-Seq) was used to screen cellular changes in local knee joint of collagen-induced arthritis (CIA) after intraarticularly injected of adiponectin. Chimera models of synovium-cartilage-NOD/SCID mice, matrigel plug assay and rat aortic ring assay were performed to demonstrate the pro-angiogenesis role of adiponectin. Cellular experiment, including proliferation, migration, apoptosis, tube formation and angiogenesis related gene expression profile, were detected with Human Umbilical Vein Endothelial Cells (HUVEC) and Mice Lung Microvessel Endothelial Cell (MLMEC) after adiponectin stimulation. Seahorse was performed to clear the influence of adiponectin to cell metabolism.

Results: The synovium and pannus hyperplasia worse in CIA model after intraarticularly injected of adiponectin, along with more serious synovitis and bone erosion. ScRNA-Seq of synovial tissues separated from CIA reminded that endothelial cell barbarically grows via metabolic remodeling after stimulated with adiponectin. Synovial chimera, matrigel plug and rat aortic ring shows adiponectin accelerates angiogenesis significantly in different background conditions. In vitro, endothelial cell proliferation detecting by RCTA and CCK8, migration by wound healing and transwell, apoptosis by FACS, tube formation and angiogenesis related gene expression profile by PCR-ARRAY were promoted by adiponectin in both HUVEC and MLMEC. Seahorse showed HUVEC made more use of glycolysis after co-cultured with adiponectin, a method of cell energy supply that tumor cells possess called warburg effect, that drives endothelial cell hyperplasia in severe environment.

Conclusion: As a classic metabolic regulator, adiponectin exacerbates CIA by promoting angiogenesis through metabolic remodeling. The findings not only provide a novel insight into the pathogenic role of adiponectin, but also reveals a potential therapeutical strategy to attenuate revascularization in RA.

Disclosure of Interests: None declared

DOI: 10.1136/annrheumdis-2021-eular.3585

\section{\begin{tabular}{|l|l}
\hline POS0399 TMT-BASED QUANTITATIVE PROTEOMICS \\
\hline
\end{tabular} ANALYSIS OF SYNOVIAL FLUID-DERIVED EXOSOMES IN RHEUMATOID ARTHRITIS, AXIAL SPONDYLOARTHRITIS, GOUT AND OSTEOARTHRITIS}

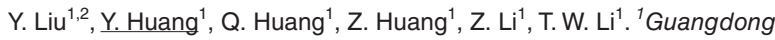
Second Provincial General Hospital, Department of Rheumatology and Immunology, Guangzhou, China; ${ }^{2}$ University of South China, Guangdong Second Provincial General Hospital, Guangzhou, China

Background: The pathogeneses of the joint diseases rheumatoid arthritis (RA) axial spondyloarthritis (axSpA), gout, and osteoarthritis (OA) are still not fully elucidated. Exosomes in synovial fluid (SF) has a critical role in the pathogenesis of arthritis. None of study has compared the proteomics of SF-derived exosomes in $\mathrm{RA}$, axSpA, gout and OA. 